\title{
Streptococcus lutetiensis Induces Autophagy via Oxidative Stress in Bovine Mammary Epithelial Cells
}

\author{
Peng Chen $\mathbb{D}^{,}{ }^{1}$ Jingyue Yang, ${ }^{1}$ Naiwen Wu, ${ }^{1}$ Bo Han, ${ }^{1}$ John P. Kastelic, ${ }^{2}$ and Jian Gao $\mathbb{D}^{1}$ \\ ${ }^{1}$ Department of Clinical Veterinary Medicine, College of Veterinary Medicine, China Agricultural University, Beijing 100193, China \\ ${ }^{2}$ Department of Production Animal Health, Faculty of Veterinary Medicine, University of Calgary, Calgary, AB, Canada T2N 4N1
}

Correspondence should be addressed to Jian Gao; gaojian2016@cau.edu.cn

Received 1 September 2021; Accepted 11 January 2022; Published 7 February 2022

Academic Editor: Ziwei Zhang

Copyright (c) 2022 Peng Chen et al. This is an open access article distributed under the Creative Commons Attribution License, which permits unrestricted use, distribution, and reproduction in any medium, provided the original work is properly cited.

\begin{abstract}
Streptococcus lutetiensis, an emerging pathogen causing bovine mastitis, has not been well characterized. We reported that $S$. lutetiensis was pathogenic both in vivo and in vitro and caused inflammatory reactions in the mammary gland. However, roles of autophagy and oxidative stress in the pathogenesis of S. lutetiensis-induced mastitis are unclear. In this study, an autophagy model of S. lutetiensis-infected bovine mammary epithelial cells (bMECs) was used to assess oxidative stress and autophagy flux. Expressions of Beclin1, light chain 3II, and Sequestosome 1/p62 were elevated in bMECs after S. lutetiensis infection. In addition, autophagosome and lysosome formation confirmed autophagy occurred. Based on LysoTracker Red and acridine orange, lysosome degradation was blocked, and lower expressions of lysosomal-associated membrane protein 2, cathepsins D, and cathepsins L confirmed lysosomal damage. Concurrently, the nuclear factor erythroid 2-related factor 2 (Nrf2), kelch-like ECH-associated protein 1 (Keap1), heme oxygenase 1 (HO1), and NAD (P)H: quinone oxidoreductase 1 (NQO1), and basilic proteins associated with the Nrf2/Keap1 signaling pathway, were detected. Decreased keap1 and increased Nrf2, HO1, NQO1, and reactive oxygen species (ROS) indicated increased oxidative stress. Treatment with N-Acetyl-L-cysteine (NAC), an ROS inhibitor, decreased both oxidative stress and autophagy. Therefore, we concluded that $S$. lutetiensis caused intracellular oxidative stress and autophagy in bMECs. In addition, crosstalk between autophagy and oxidative stress affected the autophagic flux and blocked downstream autophagy. The Nrf2-keap1-p62 pathway participated in this process, with ROS acting upstream of these effects, interfering with normal cell functions.
\end{abstract}

\section{Introduction}

Mastitis is highly prevalent in dairy cows [1], causing huge losses. Bacterial infections, often Streptococci spp., are the most common cause of mastitis [2]. S. lutetiensis, increasingly isolated from milk (Chen et al., unpublished), caused mammary gland edema and hyperemia, and we reported that bovine-derived S. lutetiensis was often resistant to multiple antibiotics [3].

Autophagy is a catabolic pathway in eukaryotic cells to remove intracellular organelles under extreme environments, to degrade damaged organelles, and control intracellular bacterial infections [4]. There are three types: macroautophagy (also called autophagy), microautophagy, and chaperone-mediated autophagy [5]. Autophagy typically involves formation of autophagosomes, fusion between autophagosomes and lysosomes, and lysosomal degradation [6]. The term "autophagic flux" includes autophagosome synthesis, delivery of autophagic substrates into the lysosome, and their subsequent degradation inside the lysosome [7]. Groups A and B Streptococci induced autophagy [8, 9]; however, the ability of group D Streptococci to induce autophagy has apparently not been reported.

An autophagosome is a double-membrane vesicle involved in macroautophagy [10]. Lysosomes are monolayer-coated vesicles containing various acidic hydrolases [11] that degrade pathogens in autolysosomes. Beclin 1 and Atgs initiate autophagy and can form complexes of several proteins that regulate autophagosome maturation and transportation [12-14]. As an essential regulator, beclin 1 ubiquitination-regulated autophagy is important in inflammation [15]. In addition, lysosomal-associated membrane 
protein 2 (LAMP2) is a major component of the lysosomal membrane [16], promoting membrane integrity and fusion of autophagy vesicles and lysosomes [17]. Cathepsins D (CTSD) and cathepsins L (CTSL) are the most abundant lysosomal proteases [18]. Lysosomes need an acidic environment, with hydrolysis promoted by $\mathrm{pH}<5.2$ [11].

Reactive oxygen species (ROS) are highly reactive oxygen-containing substances. Although ROS concentrations are well regulated under physiologic conditions, if control mechanisms are disrupted, ROS concentrations can increase and may cause damage or disease. ROS is an early inducer of autophagy during nutrient deficiency [19] and can promote degradation of ubiquitinated materials [20].

The antioxidant transcription factor, Nrf2 (nuclear factor erythroid 2-related factor 2), is activated by p62 in a "noncanonical" pathway [21]. Nrf2 is an important transcription factor regulating cellular redox homeostasis. Under physiologic conditions, Nrf2 concentration is low, whereas under oxidative stress, it is activated and translocated from the cytoplasm into the nucleus [22]; this can trigger downstream target genes responsible for detoxification and elimination of harmful substances [23]. The protein expression level of Kelch-like ECH-associated protein 1 (keap1) is a negative regulator of Nrf2 [24]. There are indications of interrelations between Nrf2 and p62 and that Nrf2 has a role in dysregulation of autophagy [25].

We reported that $S$. lutetiensis adhered to, invaded, and destroyed bovine mammary epithelial cells (bMECs) and damaged murine mammary tissue [3]. However, roles of autophagy and oxidative stress in S. lutetiensis-induced mastitis are unclear. In this study, we used an autophagy model of S. lutetiensis-infected bMECs to assess oxidative stress and autophagy flux.

\section{Materials and Methods}

2.1. Reagents and Antibodies. Acridine orange (AO) was from Solarbio. N-Acetyl-L-cysteine (NAC) was from Sigma. Bicinchoninic acid (BCA) protein assay kit and enhanced chemiluminescence kit were from Cwbio. RIPA buffer and LysoTracker Deep Red were from Beyotime Biotechnology. For these compounds, catalog information is listed in Table 1. Western blotting and immunofluorescence staining used the following primary antibodies: antiCTSL/major excreted protein and anti-CTSD from ABclonal Technology; anti-light chain 3 (LC3) B from Beyotime (Shanghai, China); anti-Sequestosome 1/p62 (SQSTM1/ p62), anti-glyceraldehyde 3-phosphate dehydrogenase (GAPDH), anti-lysosome-associated membrane protein 2 (LAMP2), anti- $\beta$-actin, anti- $\alpha$-tubulin, and anti-Beclin 1 , from Protein tech; and Peroxidase-Conjugated AffiniPure Goat Anti-mouse IgG and goat anti-rabbit IgG from Cwbio. Catalog and dilution information of these reagents are listed in Table 2.

2.2. Bacterial Strain and Cell Culture. S. lutetiensis strain was cultured in Brain Heart Infusion (BHI) broth at $37^{\circ} \mathrm{C}$ for $12 \mathrm{~h}$. After reaching $\mathrm{OD}_{600}=0.8-1.2$, bacteria were washed three times with Dulbecco's modified Eagle medium
(DMEM) without serum. The bovine mammary epithelial cell line (MAC-T) was digested with trypsin for $2 \mathrm{~min}$ at $37^{\circ} \mathrm{C}$ and centrifuged at $100 \times \mathrm{g}$ for $5 \mathrm{~min}$. The bMECs were cultured overnight at $37^{\circ} \mathrm{C}$ in $5 \% \mathrm{CO}_{2}$ without antibiotics in DMEM, supplemented with $10 \%(v / v)$ heat-inactivated fetal bovine serum (FBS) until cell density reached $80 \%$.

2.3. CCK-8 Assay. MAC-T cells ( 4,000 cells/well) were inoculated into 96-well plates. After being infected with $S$. lutetiensis $(\mathrm{MOI}=100)$, a CCK-8 kit was used to evaluate cell viability, in accordance with label protocols. After infection, $10 \mu \mathrm{l}$ of CCK-8 solution was added in each well and incubated at $37^{\circ} \mathrm{C}$ for $2 \mathrm{~h}$; finally, the absorbance was read at $490 \mathrm{~nm}$ (680 Multipurpose Microplate Reader, Bio-Rad Laboratories).

2.4. Infection of bMECs with S. lutetiensis. Autophagy caused by intracellular S. lutetiensis in bMECs was explored. Our intracellular infection model was modified from a previous study [24]. In the present study, bMECs seeded at $2 \times$ $10^{5}$ cells/well in six-well plates were infected with fresh $S$. lutetiensis grown with $\mathrm{BHI}$ broth, at multiplicity of infection (MOI) of 100 . Cells were incubated for $2 \mathrm{~h}$ at $37^{\circ} \mathrm{C}$ in $5 \% \mathrm{CO}_{2}$ to allow bacterial uptake and invasion, then washed three times with phosphate-buffered saline (PBS) and DMEM/10\% FBS with $100 \mu \mathrm{g} / \mathrm{ml}$ gentamycin (bacterial inhibiting buffer) added into each well to kill extracellular bacteria. After changing the medium to DMEM/10\% FBS with $100 \mu \mathrm{g} / \mathrm{ml}$ gentamicin, cells were incubated for the indicated intervals at $37^{\circ} \mathrm{C}$ in $5 \% \mathrm{CO}_{2}$. If not stated otherwise, inhibitors were added to incubation buffer (Figure 1(a)). To confirm the role of oxidative stress, NAC was used to pretreat cells for $3 \mathrm{~h}$, followed by exposure to S. lutetiensis $(\mathrm{MOI}=100)$ for $2 \mathrm{~h}$ and, finally, incubation in medium with $100 \mu \mathrm{g} / \mathrm{ml}$ gentamycin. The bMECs were incubated in $1 \times$ NAC from the start of NAC pretreatment to cell harvest (Figure 1(a)).

2.5. Western Blotting. For Western blot analysis, after being infected with $S$. lutetiensis for defined intervals, MAC-T cells were collected and lysed with $200 \mu \mathrm{l}$ RIPA buffer (with $1 \%$ phenylmethylsulfonyl fluoride) for $10 \mathrm{~min}$ on an ice-plate, then centrifuged at $12,000 \times \mathrm{g}$ for $15 \mathrm{~min}$ and protein concentrations quantified with a BCA protein quantity reagent kit. Equal volumes of lysates were separated by sodium dodecyl sulphate-polyacrylamide gel electrophoresis and transferred to a polyvinylidene difluoride membrane by Wet blotting. Blots were blocked in Tris-buffered saline (TBS) containing 5\% skim milk powder. Membranes were incubated with primary antibodies (Beclin 1, LC3 II/I, p62, CTSD are referred to GAPDH, and LAMP2, CTSL, Nrf2, Keap1, HO-1, and NQO- 1 are referred to $\alpha$-tubulin) at $4^{\circ} \mathrm{C}$ overnight, washed with TBS buffer with Tween 20, then incubated with secondary antibodies for $1 \mathrm{~h}$ at RT. Blotting signals were detected with an enhanced chemiluminescence Western blot detection system, images collected, and relative band density analyzed with Image J software $(\mathrm{NIH}$, Bethesda, MD, USA). 
TABLE 1: Reagent details.

\begin{tabular}{lccc}
\hline Reagent & Catalog no. & Source & City \\
\hline Acridine orange & A8120 & Solarbio & Beijing, China \\
N-acetyl-L-cysteine & A7250 & Sigma & St. Louis, MO, USA \\
Bicinchoninic acid (BCA) protein assay kit & CW0014S & Cwbio & Beijing, China \\
Enhanced chemiluminescence kit & CW0049S & Cwbio & Beijing, China \\
Ad-GFP-LC3B & C3006 & Beyotime & Shanghai, China \\
Ad-mCherry-GFP-LC3B & C3011 & Beyotime & Shanghai, China \\
LysoTracker Red & C1046 & Beyotime & Shanghai, China \\
CCK-8 & CK04 & Dojindo & Kumamoto, Japan \\
\hline
\end{tabular}

TABLe 2: Antibody details.

\begin{tabular}{lccc}
\hline Antibody & Catalog no. & Source & City \\
\hline Anti-CTSD & A13292 & ABclonal Technology & Wuhan, China \\
Anti-CTSL & A12066 & ABclonal Technology & Wuhan, China \\
Anti-LC3B & AL221 & Beyotime & Shanghai, China \\
Anti-SQSTM1/p62 & 18420 -1-AP & Proteintech & Wuhan, China \\
Anti-GAPDH & $60004-1$-Ig & Proteintech & Wuhan, China \\
Anti-LAMP2 & AF1036 & Beyotime & Shanghai, China \\
Anti- $\beta$-actin & AA128 & Beyotime & Shanghai, China \\
Anti- $\alpha$-tubulin & $66031-1-$ Ig & Proteintech & Wuhan, China \\
Anti-Beclin 1 & $11306-1-$ AP & Proteintech & Wuhan, China \\
Anti-Nrf2 & $16396-1-$ AP & Proteintech & Wuhan, China \\
Anti-keap1 & $10503-2-$ AP & Proteintech & Wuhan, China \\
Anti-NQO1 & $11451-1-$ AP & Proteintech & Wuhan, China \\
Anti-HO1 & $10701-1-$ AP & Proteintech & Wuhan, China \\
Goat anti-mouse IgG & CW0102S & Cwbio & Beijing, China \\
Goat anti-rabbit IgG & CW0103S & Cwbio & Beijing, China \\
\hline
\end{tabular}

2.6. Enzyme-Linked Immunosorbent Assay (ELISA). The superoxide dismutase (SOD) (Catalog: ml036559, mlbio, Shanghai, China), malondialdehyde (MDA) (Catalog: ml402351, mlbio), and glutathione (GSH) (Catalog: ml063357, mlbio) contents in the proteins of MAC-T cells were measured with an ELISA kit (mlbio), in accordance with label instructions.

2.7. Transient Transfection. Autophagy flux was confirmed by analyzing formation of fluorescent puncta of autophagosomes and lysosomes in GFP-LC3- or mCherry-GFP-LC3transfected cells. In this study, MAC-T cells were transfected with GFP-LC3 or mCherry-GFP-LC3 adenovirus, according to label protocols. After treatment, cells were fixed with $4 \%$ paraformaldehyde for $10 \mathrm{~min}$ and dyed with Hoechst 33342 for $5 \mathrm{~min}$ at RT. Confocal microscopy was used to examine transfected cells, and representative cells were photographed.

2.8. Transmission Electron Microscopy. To assess ultrastructure, cells were seeded on six-well plates infected with $S$. lutetiensis as described above, fixed in $4 \%$ paraformaldehyde in PBS overnight at RT, postfixed in $2 \%$ osmium tetroxide, dehydrated in a graded ethanol series (50, 70, 80, 90, and $100 \%$ for $15 \mathrm{~min}$ each), and embedded in epoxy resin.
Finally, they were kept at $37^{\circ} \mathrm{C}$ for $12 \mathrm{~h}$ and $45^{\circ} \mathrm{C}$ for $24 \mathrm{~h}$ and, finally, cured at $60^{\circ} \mathrm{C}$ for $24 \mathrm{~h}$. Ultrathin sections were prepared, stained, and examined with a Zeiss TEM 910 (Zeiss, Oberkochen, Germany).

2.9. Acridine Orange (AO) and LysoTracker Red (LTR) Staining. Cells were seeded on glass coverslips in six-well plates infected with $S$. lutetiensis, as described above. Cells were incubated in $5 \mu \mathrm{g} / \mathrm{ml} \mathrm{AO}$ or $100 \mathrm{nM} \mathrm{LTR}$ at $37^{\circ} \mathrm{C}$ for $30 \mathrm{~min}$ and fluorescence signals detected with confocal microscopy.

2.10. Measurement of Reactive Oxygen Species (ROS) Levels. Intracellular ROS generation was monitored using a fluorescence probe dichloro-dihydro-fluorescein diacetate (DCFHDA). After treatment, cells were collected, washed three times with PBS, and incubated in darkness with $100 \mu \mathrm{M}$ $\mathrm{DCFH}-\mathrm{DA}$ for $30 \mathrm{~min}$ at $37^{\circ} \mathrm{C}$. Cells were harvested, resuspended in PBS, and filtered through a $70 \mu \mathrm{m}$ filter. Flow cytometry was used to measure ROS concentrations, based on fluorescence intensity (FL-1, $530 \mathrm{~nm}$ ) of 10,000 cells. Meanwhile, the samples were detected with a fluorometric reader Spectra Max i3x (MD, America) at $488 \mathrm{~nm}$ excitation wavelength and $525 \mathrm{~nm}$ emission wavelength. 
(a1)

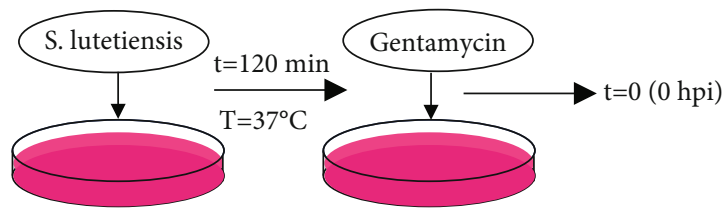

(a2)

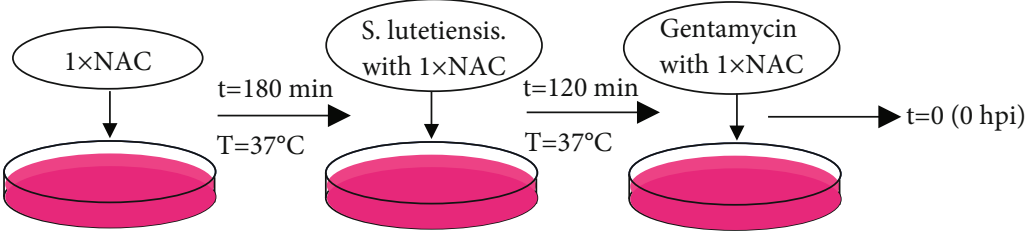

(a)

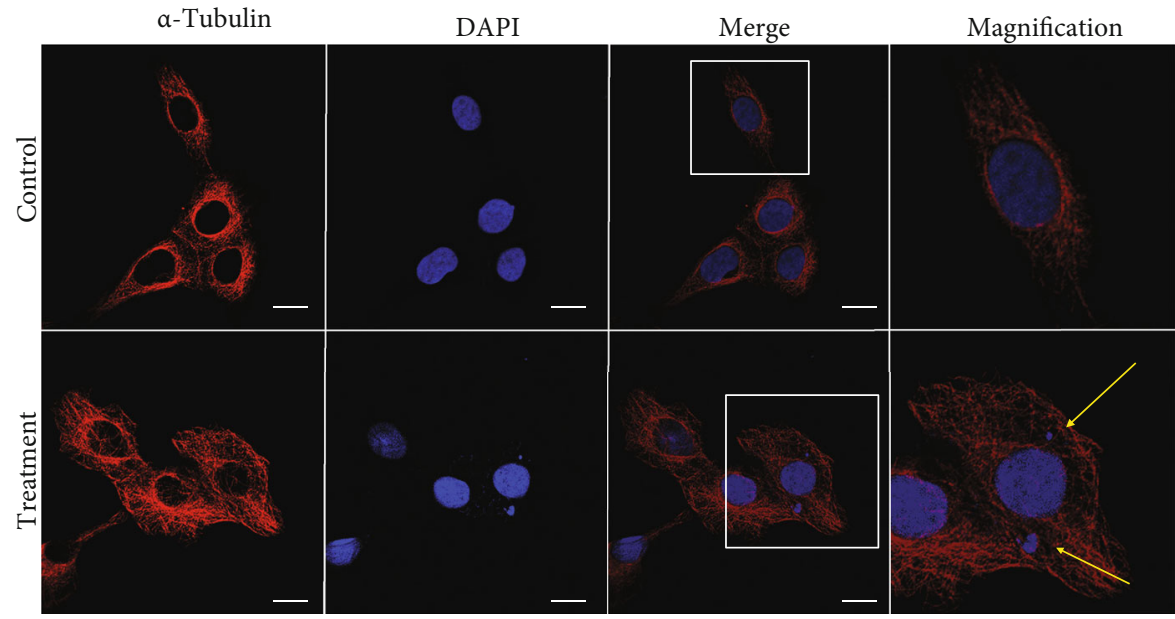

(b)

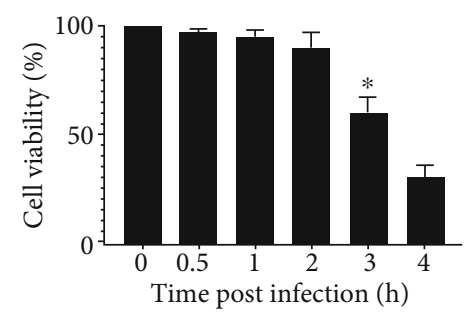

(c)

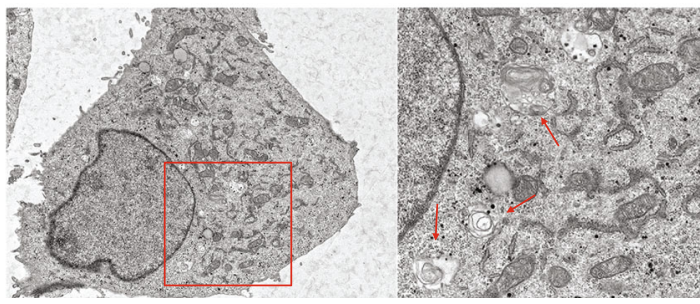

(d)

FIGURE 1: Our infection model of MAC-T cells was successful. (a) Schematic representation of our experimental design. (a1) MAC-T cells were incubated with $S$. lutetiensis for $120 \mathrm{~min}$ at $37^{\circ} \mathrm{C}$; thereafter, extracellular bacteria were killed with gentamycin. Recording of the experimental process started with time point $0 \mathrm{~h}$ postinfection (hpi). (a2) MAC-T cells were incubated with $1 \times \mathrm{NAC}$ for $180 \mathrm{~min}$ at $37^{\circ} \mathrm{C}$, then incubated with $\mathrm{S}$. lutetiensis for $120 \mathrm{~min}$ at $37^{\circ} \mathrm{C}$. Afterwards, all extracellular bacteria were killed with gentamycin. Recording of the experimental process started with time point $0 \mathrm{~h}$ postinfection (hpi). (b) Representative confocal images of invading $S$. lutetiensis and localization of tubulin with DAPI. Magnification of the outlined area, showing details; “ $\rightarrow$ ” points to S. lutetiensis. Scale bars: $10 \mu \mathrm{m}$. (c) Effects of $S$. lutetiensis invasion time on cell activity. Values are mean $\pm \mathrm{SD}, n=3,{ }^{*} p<0.05$. (d) Transmission electron microscopy. Infected for $2 \mathrm{~h}$ with S. lutetiensis; “ $\rightarrow$ ” points to S. lutetiensis inside a double-layer membrane. Scale bars: $20 \mu \mathrm{m}$.

2.11. Immunofluorescence Staining. Cells were seeded on sterile coverslips placed in 6-well plates. After cells were infected with $S$. lutetiensis for $2 \mathrm{~h}$, they were fixed in $4 \%$ paraformaldehyde for $10 \mathrm{~min}$ and then permeabilized with $0.2 \%$ Triton X-114 in PBS for 15 min. After being washed with PBS, cells were blocked with $3 \%$ BSA for $1 \mathrm{~h}$ at RT. Slides were incubated with anti- $\alpha$-tubulin antibody $(1: 200$ diluted in PBS) overnight at $4^{\circ} \mathrm{C}$ and then incubated with peroxidase-conjugated AffiniPure (diluted 1:100 in PBS) second antibody for $1 \mathrm{~h}$ at $37^{\circ} \mathrm{C}$, before nuclei and intracellular bacteria were stained with DAPI. Between steps, cells were thrice-washed with PBS. Ultimately, all slides were mounted with antifade mounting medium and examined on a Nikon A1HD25 confocal microscope with a 100x oil immersion objective. Imaging used laser wavelengths of 488 and $561 \mathrm{~nm}$. 
2.12. Statistical Analyses. All statistical analyses were performed with Statistical Product and Service Solutions 19.0 software (SPSS Inc., Chicago, IL, USA). One-way ANOVA was used to determine effects of group and time, and Duncan's multiple range test was used to locate significant differences. For all statistical analyses, $p<0.05$ was considered significant. Results are expressed as mean \pm standard deviation (SD). Data were displayed with GraphPad Prism V8.0 (Data Analysis and Graphing Software, San Diego, CA, USA).

\section{Results}

3.1. Successful Cell Infection Model. S. lutetiensis was observed in MAC-T cells of the treated group but was absent in the control group (Figure 1(b)). Based on the CCK-8 assay, continuous infection with S. lutetiensis at MOI $=100$ for $2 \mathrm{~h}$ did not decrease cell activity (Figure 1(c)). However, in ultrastructure images, $S$. lutetiensis was present in MAC-T cells, and there was an autophagic vesicle membrane structure around the bacteria (Figure $1(\mathrm{~d})$ ).

3.2. Intracellular S. lutetiensis-Induced Autophagy. Infection with $S$. lutetiensis substantially increased expression of Beclin 1 (Figure 2(a)). Furthermore, in bMECs treated with S. lutetiensis, conversion of LC3I to LC3II increased significantly (Figure 2(b)), starting from the $1^{\text {st }}$ hour postinfection (hpi) and remaining elevated for the following $3 \mathrm{~h}$, when a green fluorescent protein (GFP)-LC3 labeled autophagosome was detected by confocal imagine (Figure 2(d)). Autophagosomes (fluorescent spots) increased with interval after infection. The protein expression level of SQSTM1/p62 tended to decrease at $0.5 \mathrm{hpi}$, and then increased from 1 to 3 hpi (Figure 2(c)).

3.3. Autophagy Flux Disorder. To determine whether the autophagy flux was blocked after infection with S. lutetiensis, mCherry-GFP-LC3, which can label lysosomes, was transfected into MAC-T cells (Figure 3). Yellow fluorescence (green fluorescence merged with red fluorescence) became increasingly prominent during the postinfection period, with red mottled fluorescence peaking at $3 \mathrm{hpi}$ (Figure 3(a)), and yellow fluorescence spots quantified (Figure 3(b)).

3.4. S. lutetiensis Reduced Lysosomal $p H$. Infection with $S$. lutetiensis increased LTR staining, consistent with lysosomal acidification (Figures 4(a) and 4(b)). Red spots increased over time and accumulated around the nucleus, from 0 to $3 \mathrm{~h}$, with a modest decline at $4 \mathrm{~h}$. After staining with $\mathrm{AO}$, the color changed over time. They changed from $1 \mathrm{~h}$, peaking at $3 \mathrm{~h}$, and a slight decrease at $4 \mathrm{~h}$.

3.5. Degradation of Impaired Lysosomes. During the final stages of autophagy, autophagosomes fuse with lysosomes and are subsequently degraded. Thus, LAMP2 protein, detected via Western blotting, decreased over time (Figure 4(c)). Lysosome-related proteins CTSD and CTSL were also detected (Figures 4(d) and 4(e)). All three kinds of lysosome-associated proteins decreased over $3 \mathrm{hpi}$, indicating lysosomal degradation was impaired after S. lutetiensis infection of MAC-T.
3.6. S. lutetiensis Activated the Nrf2-Keap 1 Pathway in MAC-T Cells. Infection with S. lutetiensis decreased GSH and SOD (Figures 5(a) and 5(b)), but increased MDA (Figure 5(c)), essential indicators of oxidative stress. Regarding protein levels of Nrf2, keap1, NQO1, and HO1, S. lutetiensis increased Nrf2 protein level, whereas the Keap1 protein level was significantly below baseline (Figures 5(d) and 5(e)). HO-1 and NQO-1 are detoxifying enzymes with important roles in Nrf2-regulated phase II; protein levels of both were elevated over time in S. lutetiensis-infected cells (Figures 5(f) and 5(g)). Between 1 and $2 \mathrm{~h}$ after infection, HO-1 had a transitory decrease, whereas NQO-1 had a slight decrease at $4 \mathrm{~h}$. Therefore, the Nrf2-keap1 pathway was active when $S$. lutetiensis invaded MAC-T cells and induced oxidative stress.

3.7. ROS Contributed to S. lutetiensis-Induced Autophagy in bMECs. Oxidative stress is involved in S. lutetiensis-induced autophagy in MAC-T cells. To detect a link between ROS production and oxidative stress, ROS concentrations were assessed. Both flow cytometry analysis (Figures 6(a)-6(c)) and fluorometric reader detection (Figure 6(d)) indicated that $S$. lutetiensis increased oxidative stress and triggered excessive ROS in MAC-T cells, with increased generation of ROS infection. NAC, an inhibitor of ROS, was applied to determine whether oxidative stress was implicated in inhibition of autophagosome-lysosome fusion. Treatment with NAC significantly decreased ROS in cells infected with S. lutetiensis (Figures $7(\mathrm{a})-7(\mathrm{c})$ ). In addition, treatment for $2 \mathrm{~h}$ with $S$. lutetiensis $(\mathrm{MOI}=100)$ induced a time-dependent increase in ROS production, although the effect was abolished at $4 \mathrm{~h}$ (Figure 6(b)). In addition, NAC abolished ROS production (Figures $7(\mathrm{a})-7(\mathrm{c})$ ) and consequently oxidative stress (Figures $7(\mathrm{~d})-7(\mathrm{f})$ ), with reductions in extent of changes in GSH, SOD, and MDA. Furthermore, Nrf2, HO-1, and NQO-1 were decreased significantly compared to the treated group, whereas Keap1 protein was recovered (Figures $7(\mathrm{~g})-7(\mathrm{j})$ ). We next assessed whether ROS was involved in S. lutetiensis-induced autophagy. S. lutetiensis increased Beclin 1 protein level in bMECs, as well as the LC3 II/I ratio and p62 degradation, whereas NAC prevented these effects (Figures 8(a)-8(c)). The lysosomal protein LAMP2 was decreased after infection, but after NAC, was increased compared to the control group (Figure 8(d)). However, neither CTSD nor CTSL expression was significantly different between the treatment group and NAC-supplemented group (Figures 8(e) and 8(f)). In addition, LTR fluorescence was used to examine autophagosome-lysosome fusion (Figure $8(\mathrm{~g})$ ). These results demonstrated that increased ROS production was an upstream event contributing to S. lutetiensis activation of autophagy.

\section{Discussion}

S. lutetiensis invades cells to evade immune defenses and survives within those cells [3]. Herein, the ability to induce autophagy was achieved following optimization of S. lutetiensis dose in MAC-T cells, without compromising cell viability. This model provided clear evidence that S. lutetiensis 


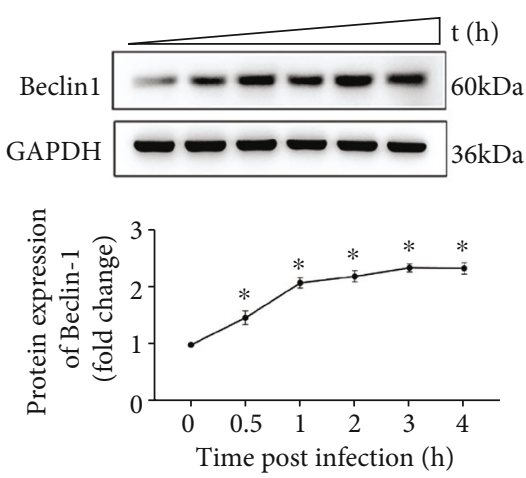

(a)
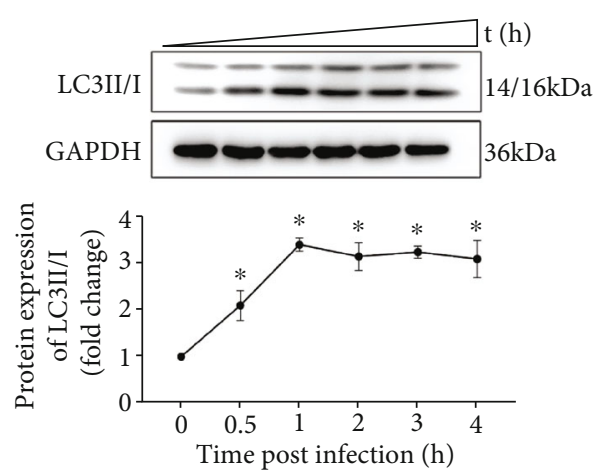

(b)
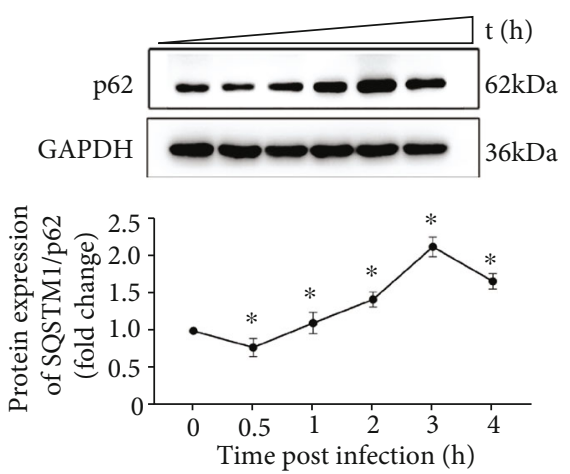

(c)

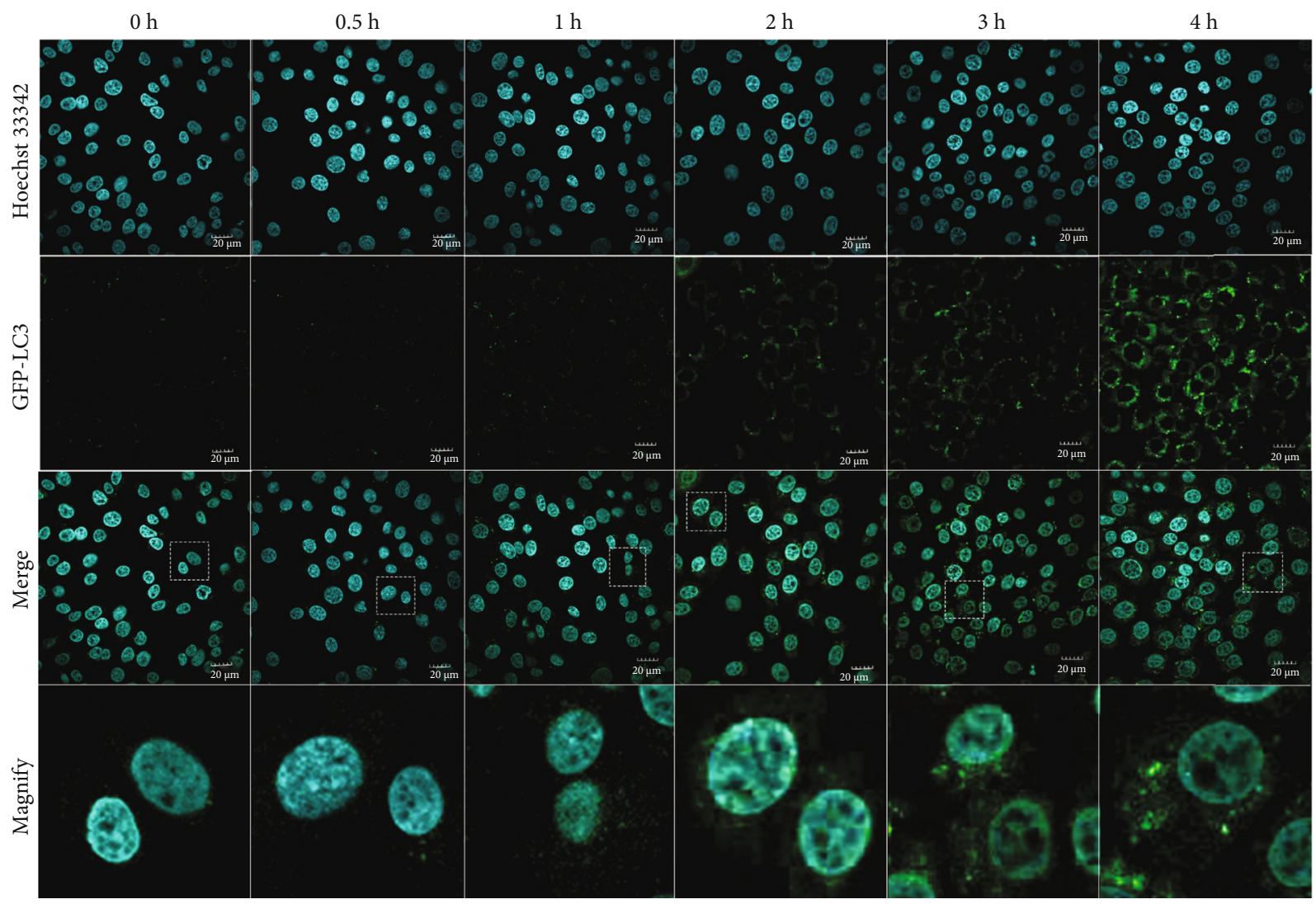

(d)

Figure 2: Intracellular S. lutetiensis-induced autophagy. (a-c) Protein level of Beclin1, LC3, and p62 in MAC-T cells with various treatments, with quantitative analysis under Western blotting bands. Data are mean $\pm \mathrm{SD}, n=3$, * represents significance with the $0 \mathrm{~h}$ group, ${ }^{*} p<0.05$. (d) Formation of GFP-LC3 puncta was observed with confocal microscopy. Scale bars: $20 \mu \mathrm{m}$.

induced autophagy in MAC-T cells by increasing oxidative stress and that there was a close relationship between oxidative stress and autophagy. Furthermore, these findings provided insights into potential mechanisms underlying $S$. lutetiensis-induced autophagy (Figure 9).

In this study, S. lutetiensis was regarded as a repelling substance; the classical vesicles of autophagosome compartments were observed and engulfed by an autophagosome, typical characteristics indicating the host activated its defense mechanism, including initiation of autophagy [26]. Moreover, Beclin 1, which is essential for both autophagy and lysosomal enzyme transport [27], was increased after infection with S. lutetiensis. Furthermore, LC3 II, an autoph- agy marker with levels related to the intensity of autophagy [28], uses GFP-LC3 for confirming autophagy accumulation [29]. Therefore, expression of LC3 II was assessed by GFPLC3 detected with confocal microscopy after MAC-T cells were infected with $S$. lutetiensis, with a time-independent tendency for green spots. Collectively, all of these outcomes indicated that $S$. lutetiensis induced autophagy.

SQSTM1/p62 acts as a relative protein to mediate degradation of its recognition substrate $[30,31]$. Excessive buildup of SQSTM1/p62 sequestered keap1, an adaptor of the E3-ubiquitin ligase complex for Nrf2 [32]. In the present study, expression of SQSTM1/p62 protein began to rise from the second time point $(0.5 \mathrm{~h}$ after infection) during $S$. 


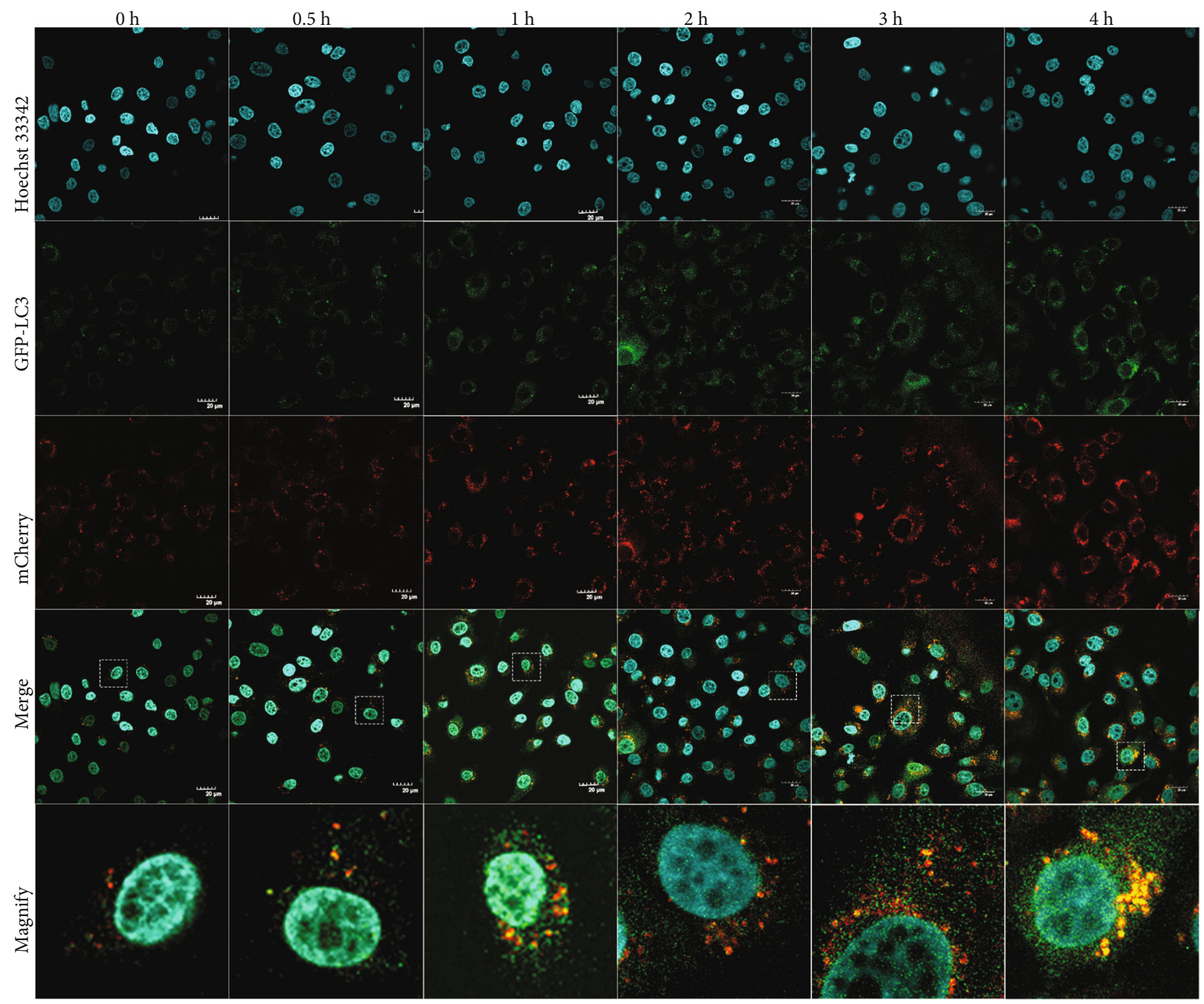

(a)

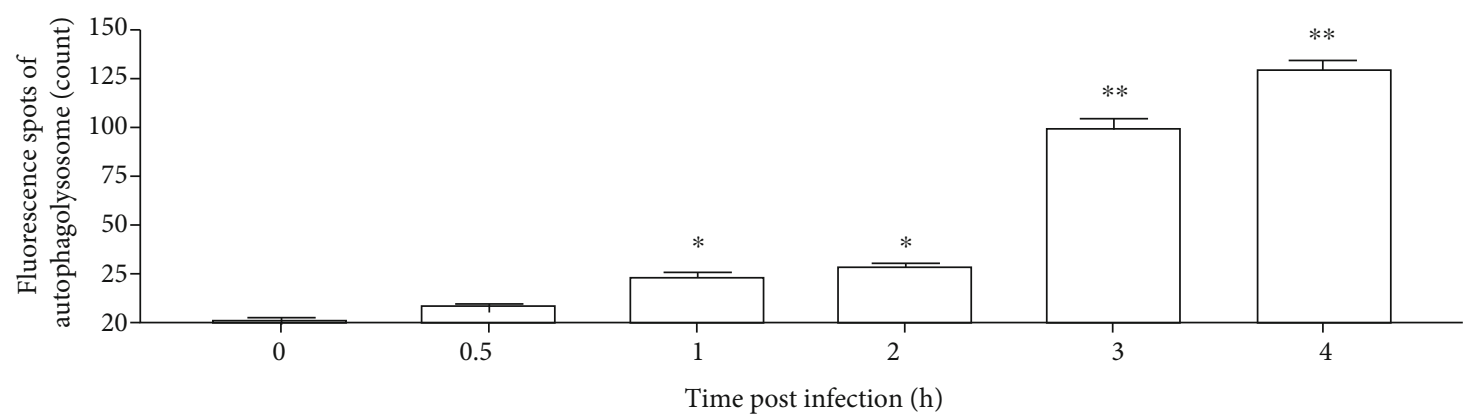

(b)

Figure 3: Autophagy flux disorder. Cells were grown on coverslips and transfected with mCherry-GFP-LC3 at MOI $=20$ for $24 \mathrm{~h}$, then infected with S. lutetiensis for various intervals to monitor the autophagic flux; (a) representative confocal images. Scale bars: $20 \mu \mathrm{m}$. (b) Quantity of autophagolysosome (mean $\pm \mathrm{SD}, n=3,{ }^{*}$ represents the significance between adjacent time points, ${ }^{*} p<0.05,{ }^{* *} p<0.01$ ).

lutetiensis infection in bMECs. Meanwhile, expression of Nrf2 increased, whereas keap1 decreased from 0 to $3 \mathrm{hpi}$, consistent with previous studies [24]. The mCherry-GFPLC3 is an adenovirus probe to detect the rate of autophagic flux [33]. In this study, accumulation of yellow fluorescence was observed by mCherry-GFP-LC3 after S. lutetiensis infection, indicating that the autophagic flux was blocked at fusion of autophagosomes and lysosomes. In addition, $S$. lutetiensis induced increases in the LC3 II/I ratio and p62 degradation, providing further support for autophagy 


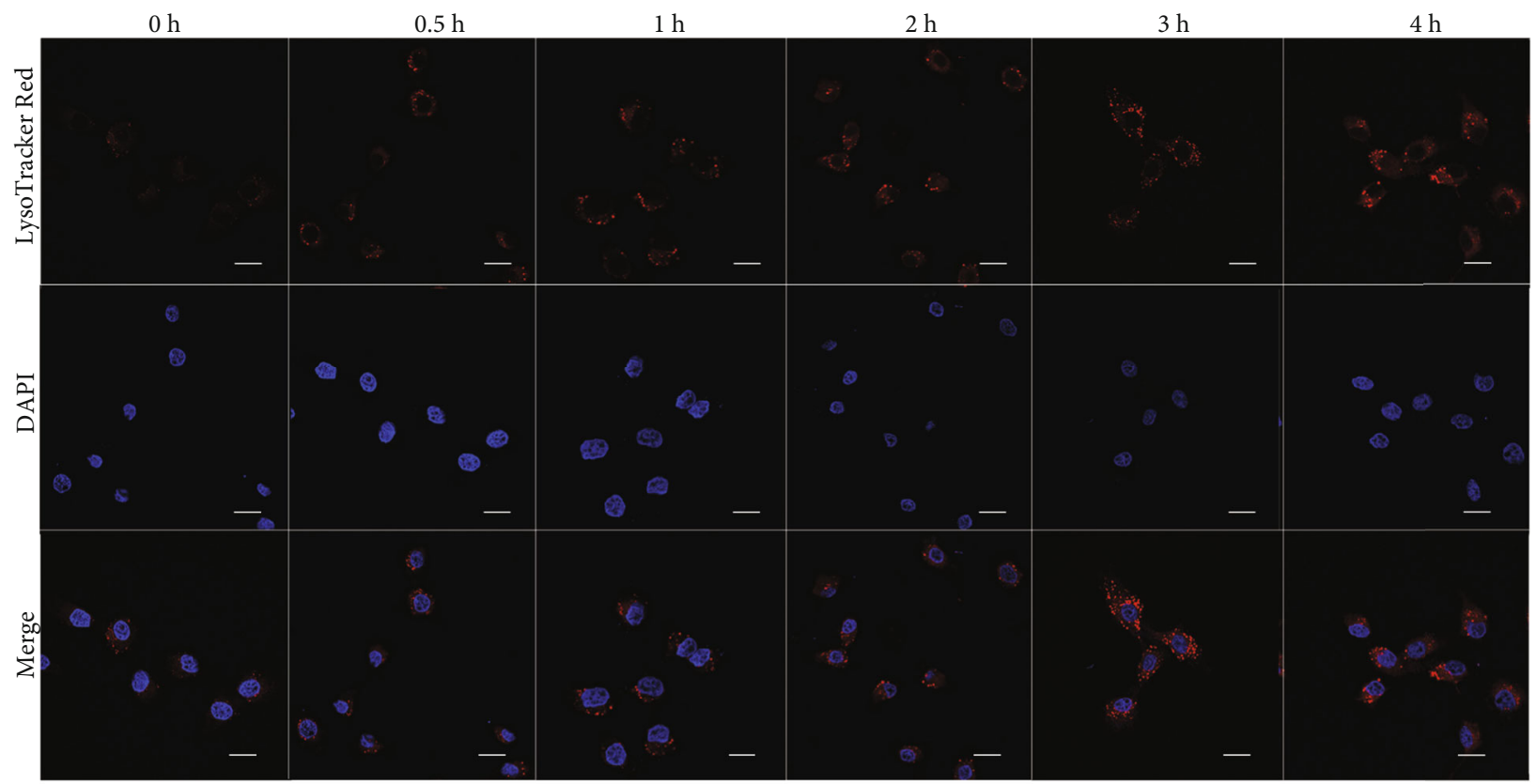

(a)

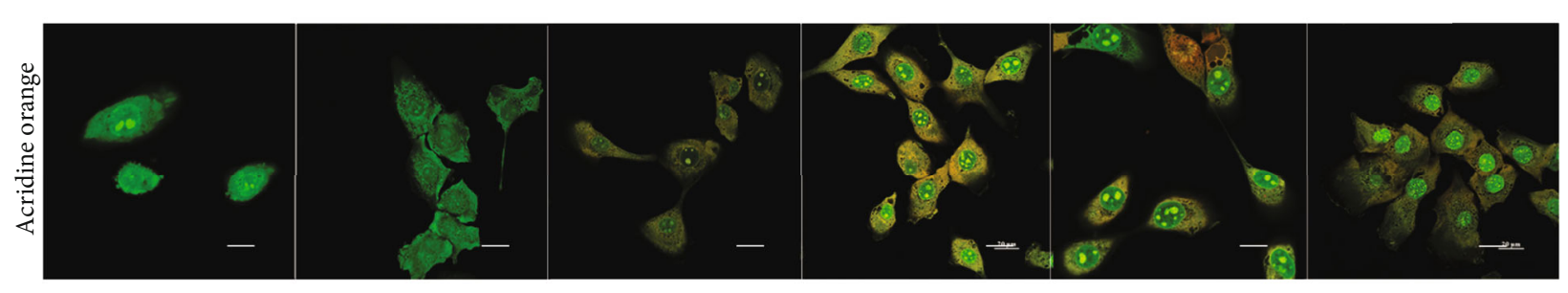

(b)
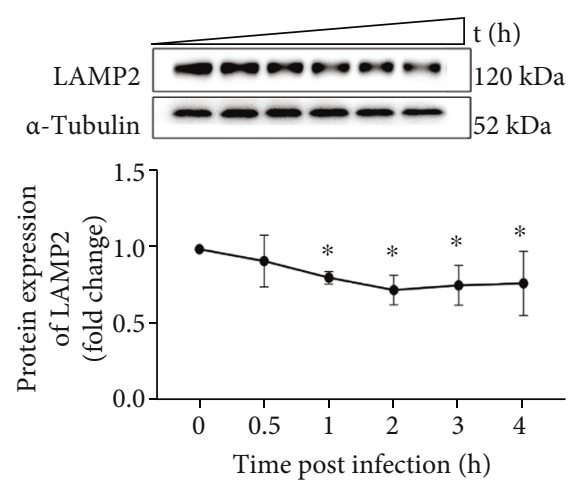

(c)

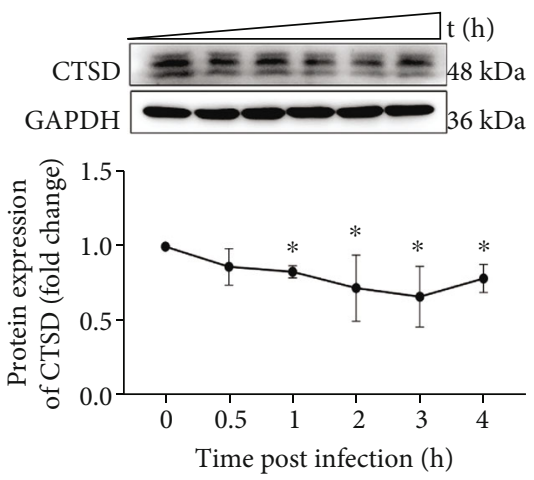

(d)
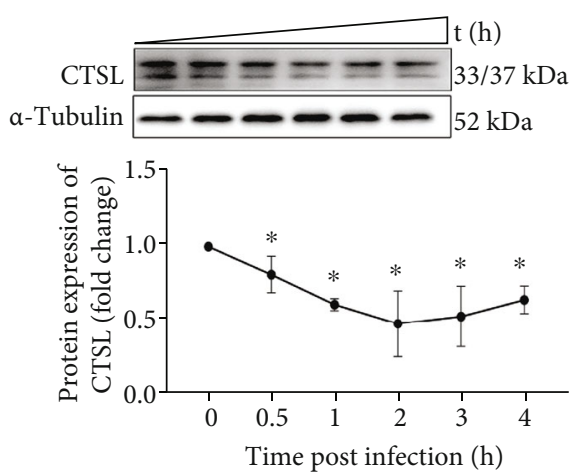

(e)

FIgURE 4: S. lutetiensis decreased $\mathrm{pH}$ in lysosomes to further block the autophagy flux. (a) To assess lysosomal $\mathrm{pH}$, cells were stained with $100 \mathrm{nM}$ LysoTracker Deep Red at $37^{\circ} \mathrm{C}$ for $30 \mathrm{~min}$ after being infected with S. lutetiensis. Scale bars: $20 \mu \mathrm{m}$. (b) After being infected with S. lutetiensis, cells were stained with $\mathrm{AO}$ at $37^{\circ} \mathrm{C}$ to assess lysosomal pH. Scale bars: $20 \mu \mathrm{m}$. (c-e) Protein levels of LAMP2, cathepsin D, and cathepsin L in MAC-T cells at various intervals after infection with S. lutetiensis. Upper panels: representative Western blot images; lower panels: quantitative analysis (mean $\pm \mathrm{SD}, n=3$, ${ }^{*}$ represents the significance with the 0 h group, ${ }^{*} p<0.05$ ).

induction. Moreover, LAMP2 labelling, as well as cell staining with AO and LTR, demonstrated that S. lutetiensis weakened lysosomal activity.

Autophagy formation is the first step of autophagy flux. In this step, autophagosomes can fuse with lysosomes or late endosomes and then fuse with lysosomes [34]. Lysosomal degradation is an essential factor for autophagy flux, and a slightly acidic environment for lysosomes promotes proteolytic enzyme degradation of organelles [35]. LTR is a reliable probe to detect changes in intercellular $\mathrm{pH}$, as it accumulates in an acidic environment, with the intensity of red fluorescence corresponding to the $\mathrm{pH}[36,37]$. In addition, $\mathrm{AO}$ is a specific probe that can be trapped in lysosomes [38]. In the present study, the intensity of LTR peaked at $3 \mathrm{~h}$ after 


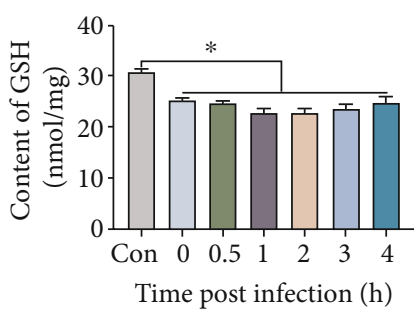

(a)

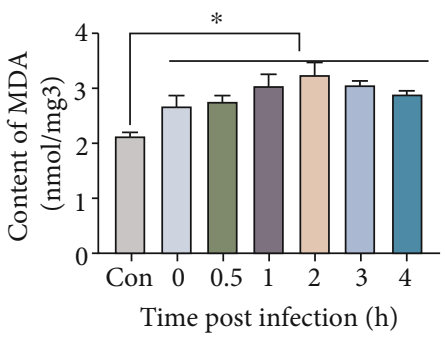

(c)

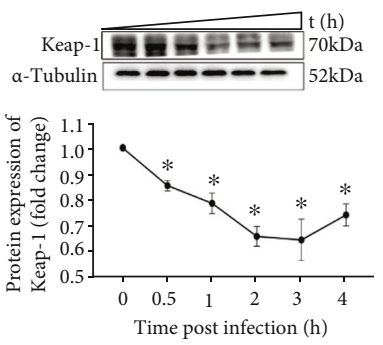

(e)

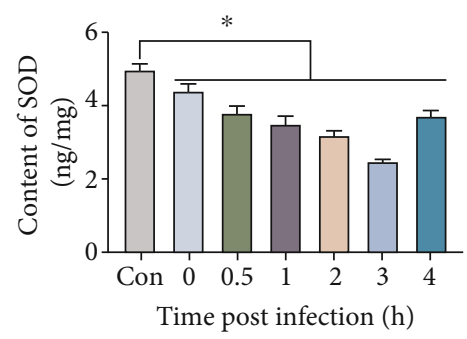

(b)

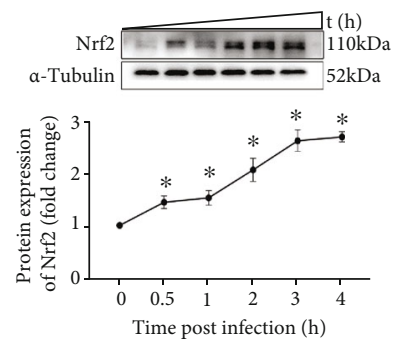

(d)

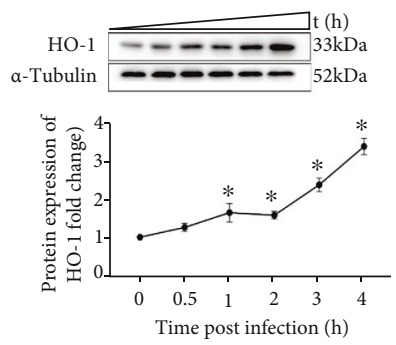

(f)

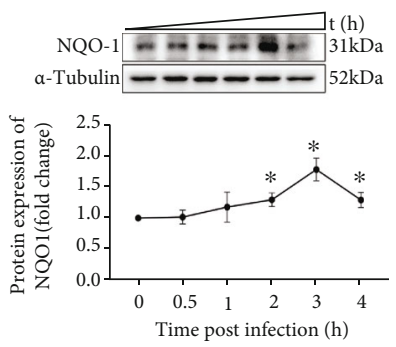

(g)

FIGURE 5: S. lutetiensis induce oxidative stress in MAC-T cells. (a-c) Protein levels of GSH, SOD, and MDA detected by ELISA. (d-g) Protein levels of Nrf2, keap1, HO-1, and NQO-1 in MAC-T cells at various intervals after infection with S. lutetiensis. Upper panels: representative Western blot images; lower panels: quantitative analysis (mean $\pm \mathrm{SD}, n=3,{ }^{*}$ represents the significance with $0 \mathrm{~h}$ group, $\left.{ }^{*} p<0.05\right)$.

S. lutetiensis infection, with AO staining having a similar outcome. These two approaches both provided evidence that lysosomes accumulated due to a blockage of the downstream autophagy flux, which differed from a report that group A Streptococcus was efficiently killed within a lysosome-fused autophagosome compartment [9].

Cathepsins are the major lysosomal proteases involved in autophagic degradation, wherein CTSD and CTSL are two abundant lysosomal proteases [39]. When activation of cathepsin proteases requires acidification, the altered $\mathrm{pH}$ decreased protein degradation [40]. CTSD is involved in intracellular catabolism in lysosomal compartments [41]; by activating CTSB, CTSL can hydrolyze proteins, hormones, and phagocytic bacteria [42]. In this study, expression of CTSD and CTSL decreased initially, but subsequently recovered to a basal level, indicating that intracellular invasion of S. lutetiensis had a negative effect on lysosomes, and cathepsins had self-healing ability.

As an essential mechanism for eliminating damaged organelles and exogenous foreign body, autophagy is regulated by various cellular process such as nutrient deficiencies, oxidative stress, and other influencing factors [43]. Autophagy and oxidative stress in a S. lutetiensis-infected model have apparently not been reported. Herein, we determined that oxidative stress had an essential role in S. lutetiensisinduced cyto-injury. As a transcription factor, Nrf2 regulates 


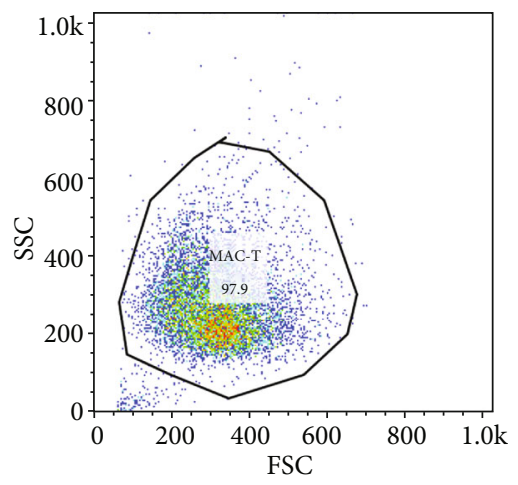

(a)
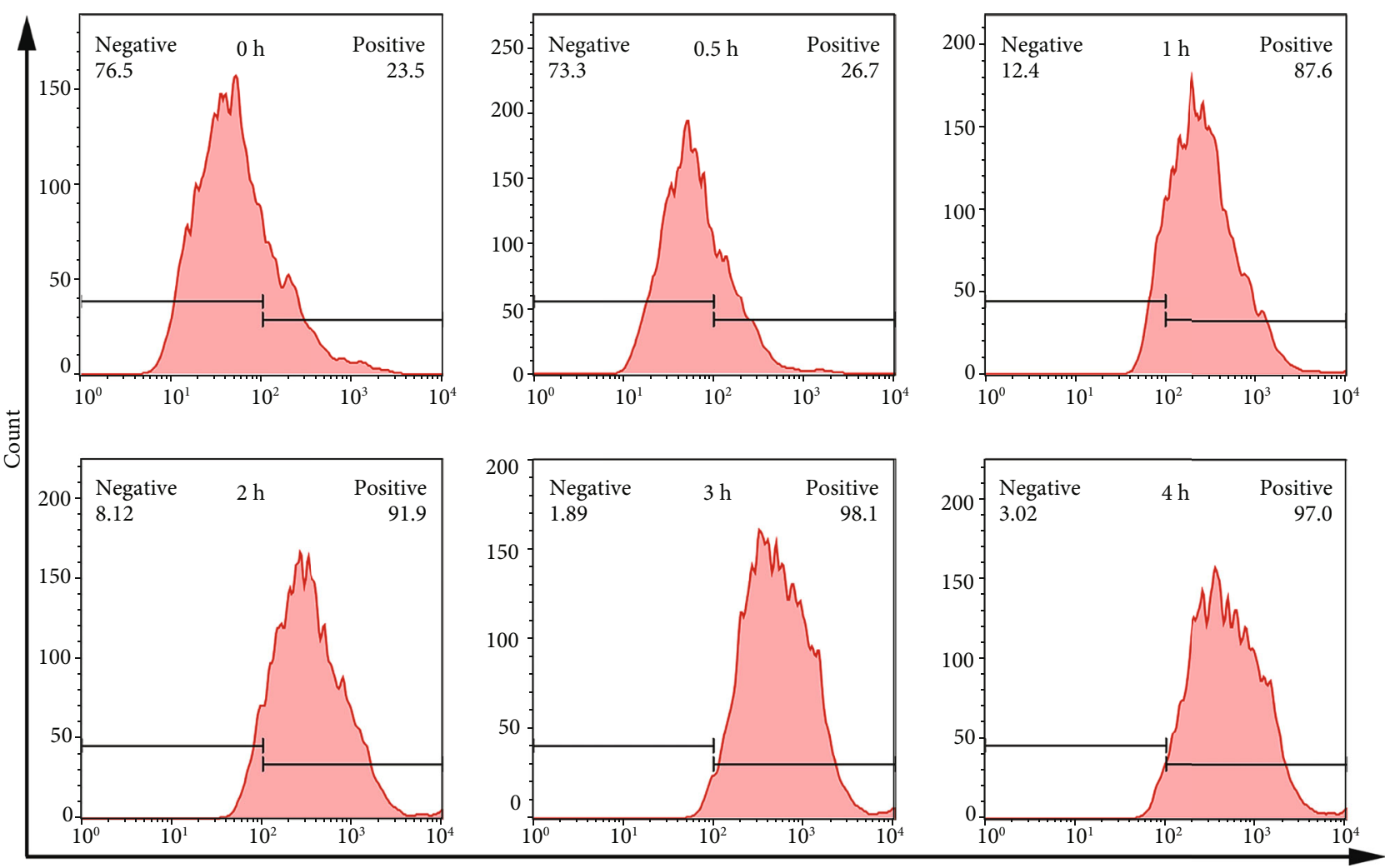

DCF-fluorescence

(b)

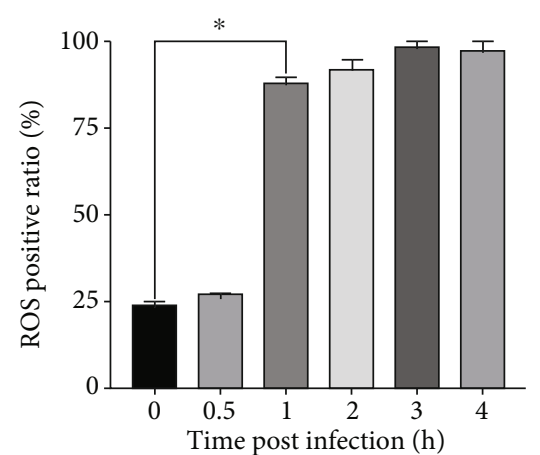

(c)

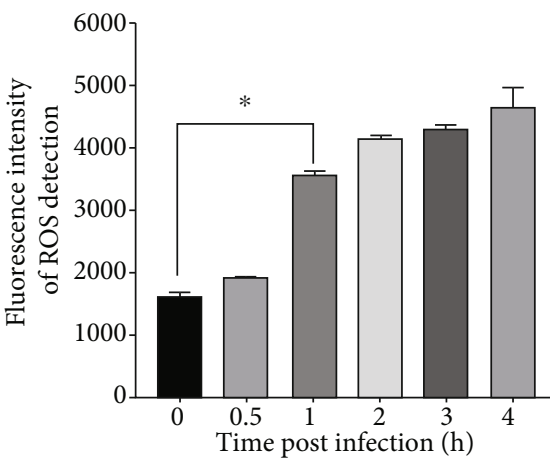

(d)

FIGURE 6: ROS expression in MAC-T cells at various intervals after infection with S. lutetiensis (a-c) ROS detected by flow cytometry. (d) Fluorescence intensity of ROS detected by fluorometric reader (mean $\pm \mathrm{SD}, n=3,{ }^{*}$ represents the significance with $0 \mathrm{~h}$ group, ${ }^{*} p<0.05$ ). 


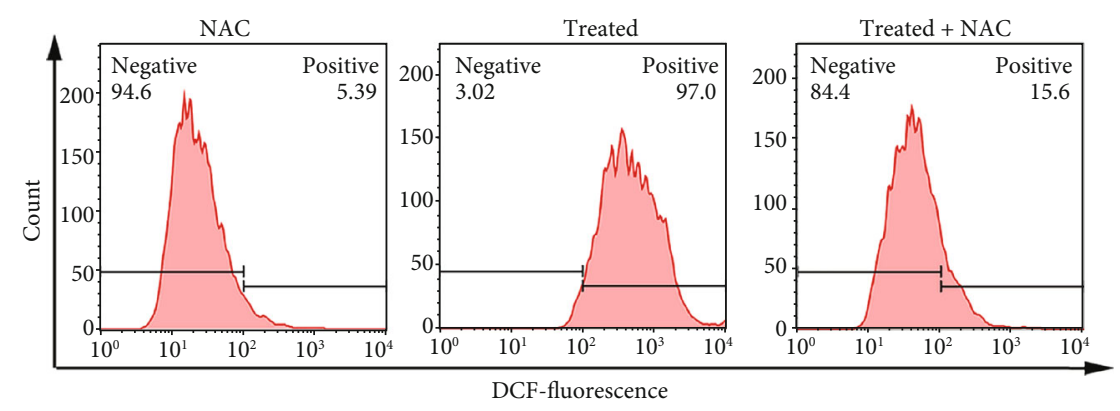

(a)

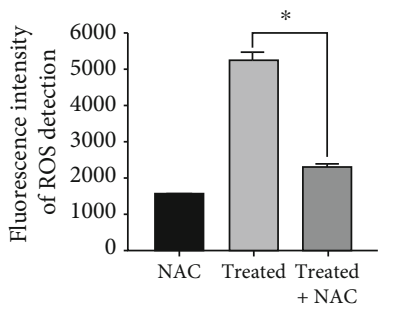

(c)

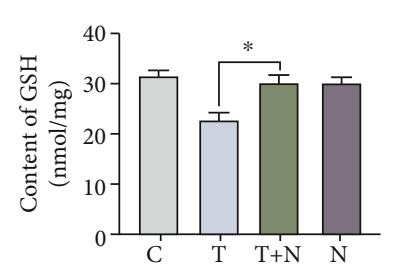

(d)

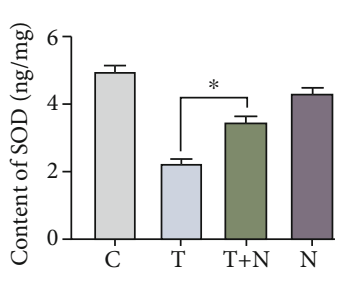

(e)

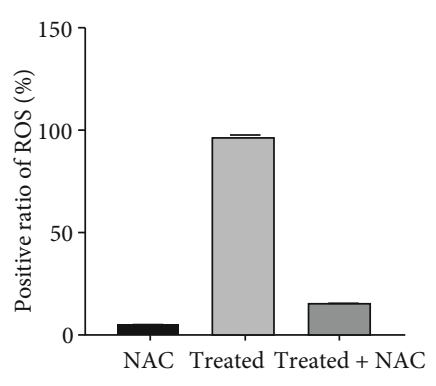

(b)

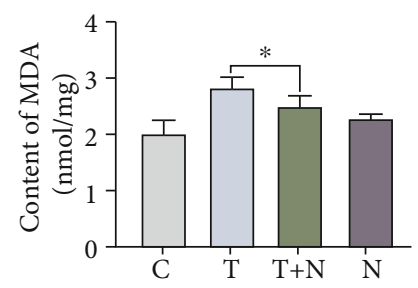

(f)

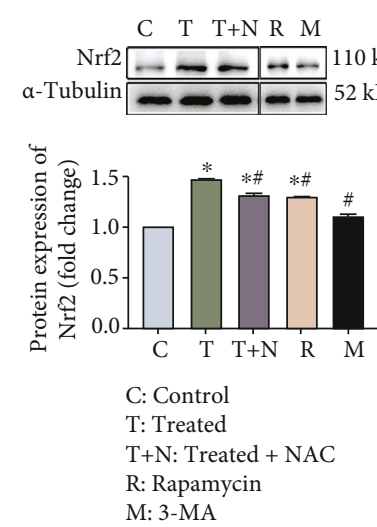

(g)
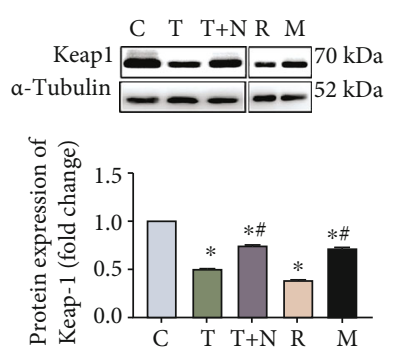

\section{C: Control \\ T: Treated \\ $\mathrm{T}+\mathrm{N}$ : Treated + NAC \\ R: Rapamycin}

M: 3-MA

(h)
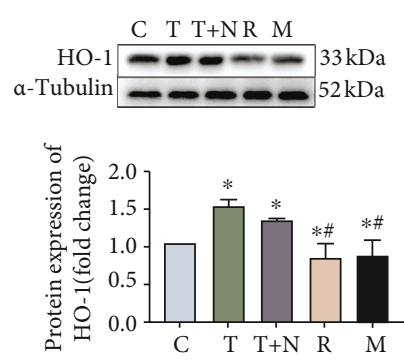

C: Control

T: Treated

$\mathrm{T}+\mathrm{N}:$ Treated $+\mathrm{NAC}$

R: Rapamycin

M: 3-MA

(i)

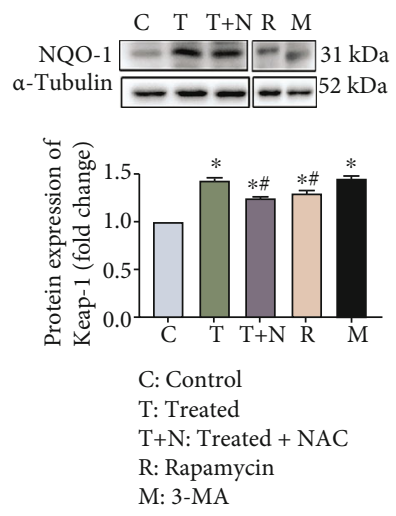

(j)

Figure 7: NAC efficiently decreased S. lutetiensis-induced oxidative stress in MAC-T. (a-c) The difference of ROS expression in MAC-T cells between treated with $S$. lutetiensis and treated with NAC (detected by flow cytometry and fluorometric reader). (d-f) Protein levels of GSH, SOD, and MDA detected by ELISA. (g-j) Protein levels of Nrf2, keap1, HO-1, and NQO-1 in MAC-T cells after various treatments. Upper panels: representative Western blot images; lower panels: quantitative analysis (mean $\pm \mathrm{SD}, n=3$, * represents the significance with the control group, ${ }^{*}$ represents the significance with the treated group, ${ }^{*} p<0.05,{ }^{\#} p<0.05$; C: control; T: treatment; $\mathrm{T}+\mathrm{N}$ : treatment + NAC; R: rapamycin; $\mathrm{M}: 3-\mathrm{MA})$. 

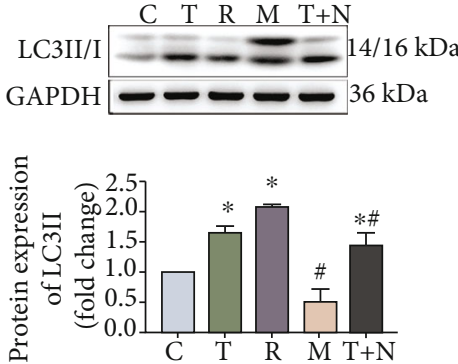

C: Control

$\mathrm{T}$ : Treatment

R: Rapamycin

M:3-MA

$\mathrm{T}+\mathrm{N}$ : Treatment+NAC

(a)
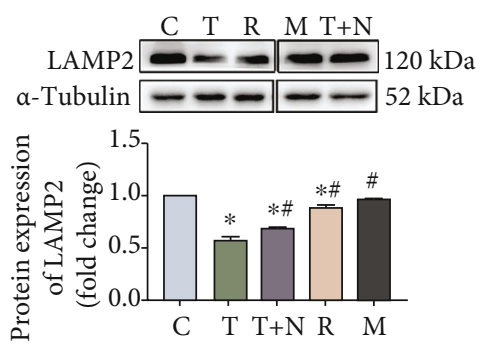

C: Control

$\mathrm{T}$ : Treatment

R: Rapamycin

M:3-MA

$\mathrm{T}+\mathrm{N}$ : Treatment+NAC

(d)
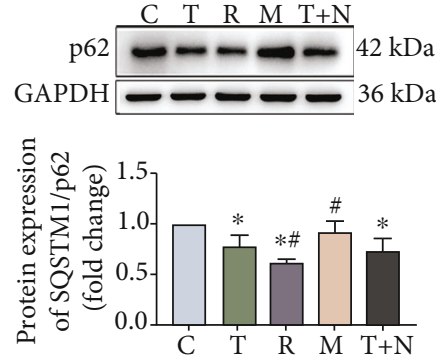

C: Control

T: Treatment

R: Rapamycin

M:3-MA

$\mathrm{T}+\mathrm{N}$ : Treatment $+\mathrm{NAC}$

(b)

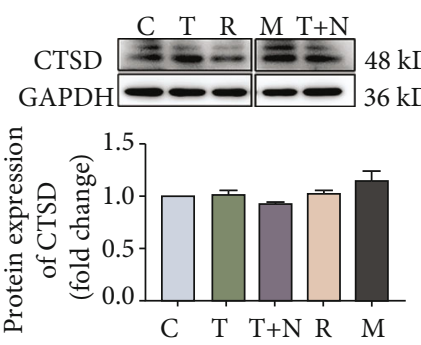

C: Control

T: Treatment

R: Rapamycin

M:3-MA

$\mathrm{T}+\mathrm{N}$ : Treatment+NAC

(e)
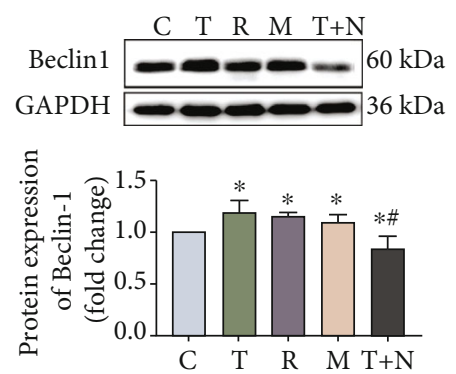

C: Control

T: Treatment

R: Rapamycin

M:3-MA

$\mathrm{T}+\mathrm{N}$ : Treatment+NAC

(c)

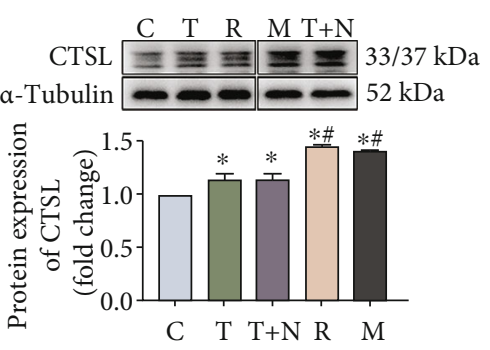

C: Control

$\mathrm{T}$ : Treatment

R: Rapamycin

M:3-MA

$\mathrm{T}+\mathrm{N}$ : Treatment+NAC

(f)

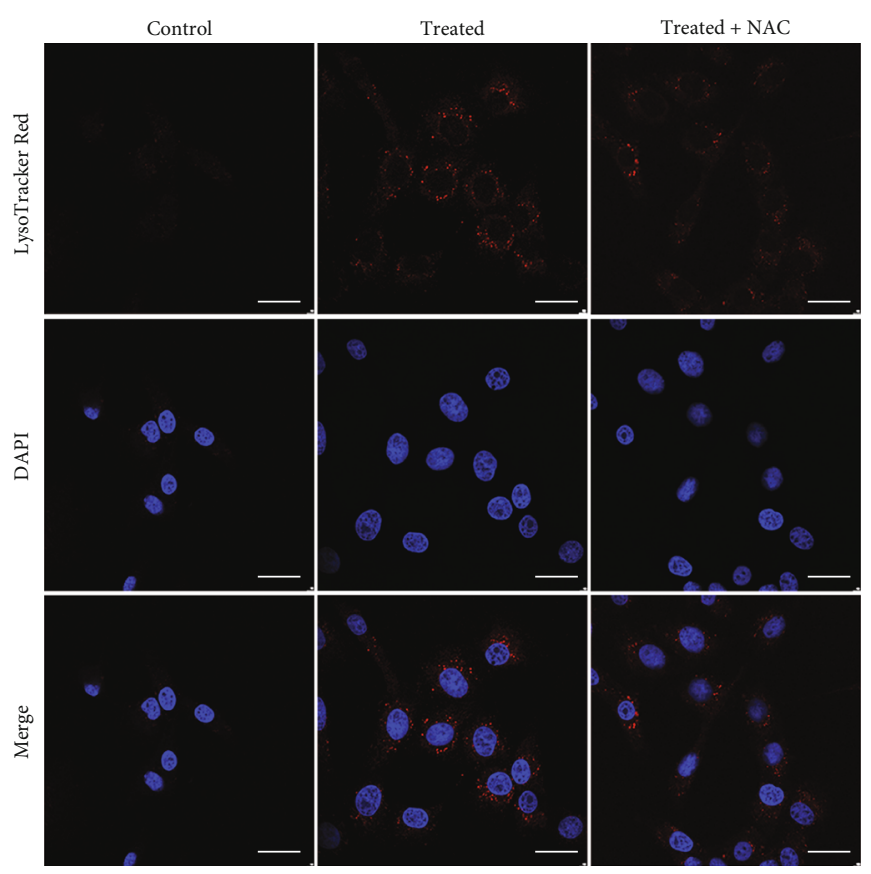

(g)

FIgURE 8: NAC efficiently decreased S. lutetiensis-induced autophagy in MAC-T. (a-f) Protein levels of LC3II/I, p62, Beclin1, LAMP2, CTSD, and CTSL. Upper panels: representative Western blot images; lower panels: quantitative analysis (mean \pm SD, $n=3,{ }^{*}$ represents the significance with the control group, ${ }^{\#}$ represents the significance with the treated group, ${ }^{*} p<0.05,{ }^{*} p<0.05$; C: control; T: treatment; $\mathrm{R}$ : rapamycin; M: 3-MA; T+N: treatment + NAC). (g) Lysosome detection comparison between infected with $S$. lutetiensis with/without NAC. Scale bars: $20 \mu \mathrm{m}$. 


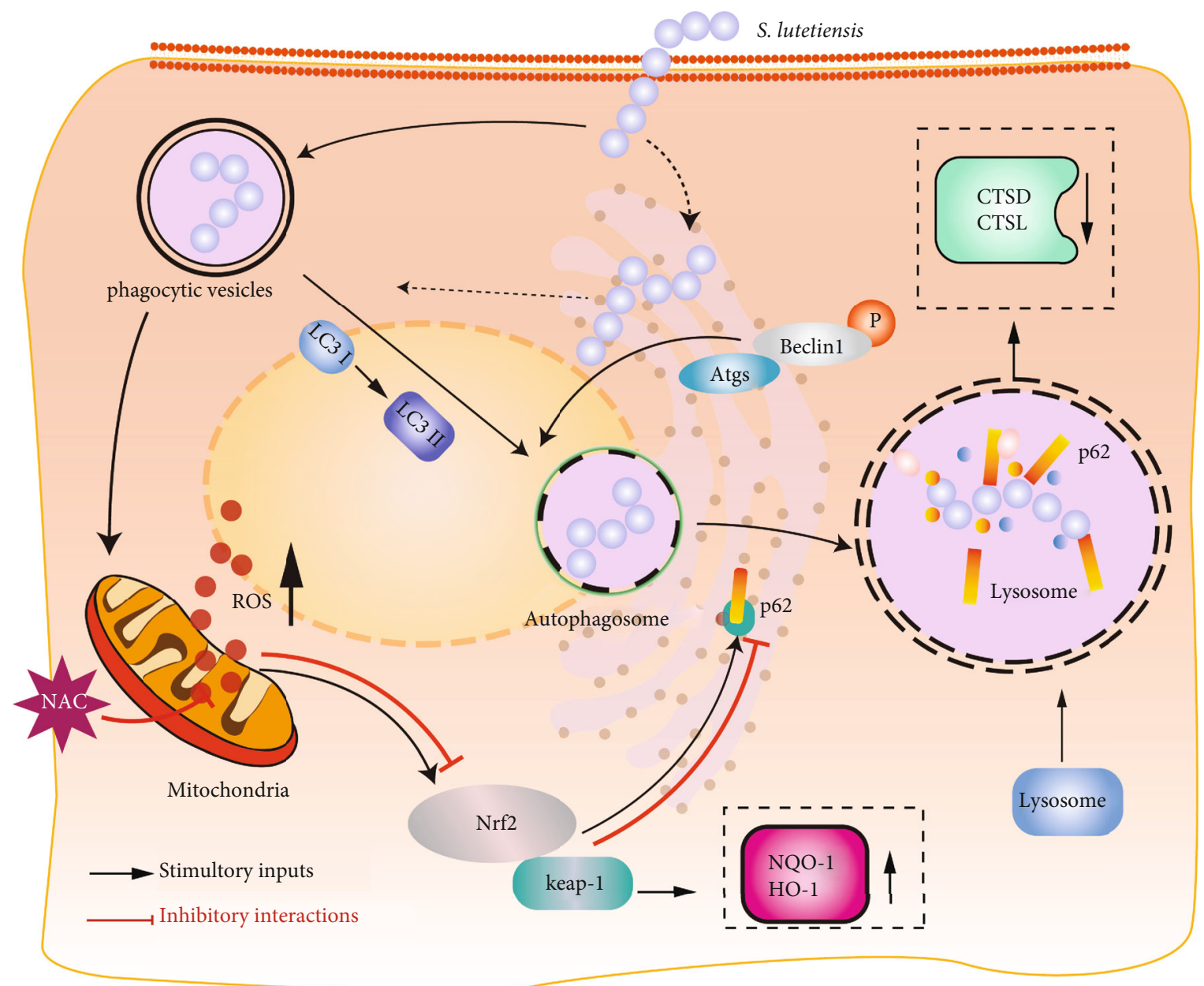

Figure 9: Putative signal pathway of S. lutetiensis inducing autophagy in bovine mammary gland epithelia though oxidative stress. Schematic diagram illustrating that $S$. lutetiensis can invade MAC-T cells and lead to autophagy by stimulating mitochondrial oxidative stress. In this process, the ROS-Nrf2-keap1 pathway has an essential role. ROS is upstream of these effects. Black arrows and red bars indicate stimulation and inhibition, respectively.

a cluster of oxidative stress-inducible genes in cells. There is considerable evidence that the Nrf2-Keap1 antioxidant system has an important cytoprotective defense mechanism in alleviating oxidative insults $[44,45]$. Meanwhile, Keap1 can indirectly assess activation of Nrf2, as the Nrf2-Keap1 pathway is a regulator for the endogenous antioxidant response [46]. Therefore, Nrf2-Keap1 system integrity is maintained by an autophagy pathway [47], and activation of $\mathrm{Nrf} 2$ is prolonged if autophagy is disrupted [25]. In response to oxidative stress, Nrf2 transcription factor could induce expression of p62 [48, 49]. Our study implied that transportation of oxidized proteins to autophagosomes for degradation can decrease oxidative injury. In response to oxidative stimuli, keap1 lost its ability to bind with Nrf2. Therefore, we speculate that Nrf2 may enter the nucleus and bind to the antioxidant response element (ARE), to further regulate downstream genes $\mathrm{HO}-1$ and NQO-1, as they are key oxidative stress indicators regulated by $\mathrm{Nrf} 2$ [24]. Furthermore, HO-1 and NQO-1 proteins in MAC-T cells were elevated after $S$. lutetiensis infection. Recent research demonstrated that NAC could block ROS production and further reversed LC3 II accumulation which trig- gered by coenzyme $\mathrm{Q}_{0}$, and the same mechanism of NAC also occurred in chrysin-induced autophagy [50, 51]. We used NAC, an efficient ROS inhibitor [52, 53]. Modulation of antioxidant enzymes (SOD, GSH, and MDA) indicated inhibition of oxidative stress. Meanwhile, expression of NQO-1 and HO-1 was restored to basal levels; therefore, we inferred that Nrf2 may be a key cytokine in oxidative stress and autophagy.

As arguably the most influential intracellular signaling molecule, ROS regulates cell function and promotes homeostasis [54]. There are indications that oxidative stress can be a vital stimulus, via regulation of ROS, to stimulate autophagy $[21,55]$. Autophagy and oxidative stress have an intricate relationship in many diseases [26]; hyperthermia can enhance both autophagy and ROS generation, implying potential associations between autophagy and oxidative stress [56]. Moreover, several studies reported the relationship between oxidative damage and autophagy [57], although this has apparently not been reported in bMECs. In this study, ROS were rapidly produced after exposure to $S$. lutetiensis. Notably, after $2 \mathrm{~h}, \mathrm{~S}$. lutetiensis induced accumulation of LC3II and degradation of p62, suggesting 
that ROS generation preceded initiation of autophagy. Furthermore, NAC effectively blocked autophagy activation by $S$. lutetiensis. As a main component of oxidative stress, ROS could be the link between autophagy and oxidative stress in bMECs.

The current study, apparently the first investigation of the role between autophagy and oxidative stress induced by S. lutetiensis, identified crosstalk between autophagy and oxidative stress. The limitation of this study was that an in vitro bMEC infection model was used to evaluate autophagy and oxidative stress. In future studies, we plan to investigate whether similar interactions occur in vivo in mammary tissue.

\section{Conclusions}

We characterized autophagy induced by oxidative stress in bMECs infected with $S$. lutetiensis isolated from bovine mastitis. We concluded that S. lutetiensis induced autophagy of bMECs by upregulating oxidative stress, although lysosomes accumulated due to a blockage of the downstream autophagy flux. In this process, there was crosstalk between autophagy and oxidative stress; the latter affected autophagy by intervening in the Nrf2-keap1-p62 pathway, with ROS acting upstream of these effects.

\section{Data Availability}

All data generated or analyzed during this study are included in this published article.

\section{Ethical Approval}

This work had received approval for research ethics from the Ethical Committee of the College of Veterinary Medicine, China Agricultural University (CAU), Beijing. Furthermore, this study was conducted according to standard ethical guidelines implemented at CAU (SYXK, 2016-0008), and a certificate of approval is available upon request.

\section{Conflicts of Interest}

The authors declare they have no actual or potential competing financial interests.

\section{Acknowledgments}

This study was supported financially by the following: Ningxia Key R\&D Project (no. 2019BBF02027), Hebei Key R\&D Project (19226607D), Beijing Municipal Natural Science Foundation (no. 6192013), the National Natural Science Foundation of China (no. 31772813), and the High-End Foreign Experts Recruitment Program (no. GDT20171100013).

\section{References}

[1] X. Y. Hu, S. M. Li, Y. H. Fu, and N. S. Zhang, "Targeting gut microbiota as a possible therapy for mastitis," European Journal of Clinical Microbiology \& Infectious Diseases, vol. 38, no. 8, pp. 1409-1423, 2019.
[2] J. Gao, H. W. Barkema, L. Zhang et al., "Incidence of clinical mastitis and distribution of pathogens on large Chinese dairy farms," Journal of Dairy Science, vol. 100, no. 6, pp. 47974806, 2017.

[3] P. Chen, Y. Qiu, G. Liu et al., "Characterization of Streptococcus lutetiensis isolated from clinical mastitis of dairy cows," Journal of Dairy Science, vol. 104, no. 1, pp. 702-714, 2021.

[4] W. Hu, H. Chan, L. Lu et al., "Autophagy in intracellular bacterial infection," Seminars in Cell \& Developmental Biology, vol. 101, pp. 41-50, 2020.

[5] M. Andrade-Tomaz, I. de Souza, C. R. R. Rocha, and L. R. J. C. Gomes, "The role of chaperone-mediated autophagy in cell cycle control and its implications in cancer," Cell, vol. 9, no. 9, p. 2140, 2020.

[6] M. Yamamoto, S. O. Suzuki, and M. Himeno, "The effects of dynein inhibition on the autophagic pathway in glioma cells," Neuropathology, vol. 30, no. 1, pp. 1-6, 2010.

[7] D. J. Klionsky, K. Abdelmohsen, A. Abe et al., "Guidelines for the use and interpretation of assays for monitoring autophagy ( $3^{\text {rd }}$ edition)," Autophagy, vol. 12, no. 1, pp. 1-222, 2016.

[8] M. Ogawa, N. Takada, S. Shizukuishi et al., "Streptococcus pneumoniae triggers hierarchical autophagy through reprogramming of LAPosome-like vesicles via NDP52-delocalization," Communications Biology, vol. 3, no. 1, 2020.

[9] Y. L. Cheng, Y. W. Wu, C. F. Kuo et al., "Galectin-3 inhibits Galectin-8/Parkin-mediated ubiquitination of group A Streptococcus," mBio, vol. 8, no. 4, 2017.

[10] V. Roca-Agujetas, C. de Dios, L. Lestón, M. Marí, A. Morales, and A. Colell, "Recent insights into the mitochondrial role in autophagy and its regulation by oxidative stress," Oxidative Medicine and Cellular Longevity, vol. 2019, 16 pages, 2019.

[11] M. Fukuda, "Biogenesis of the lysosomal membrane," Sub-Cellular Biochemistry, vol. 22, pp. 199-230, 1994.

[12] B. Ma, W. Cao, W. Li et al., "Dapper1 promotes autophagy by enhancing the Beclin1-Vps34-Atg14L complex formation," Cell Research, vol. 24, no. 8, pp. 912-924, 2014.

[13] E. Itakura and N. Mizushima, "Atg14 and UVRAG: mutually exclusive subunits of mammalian Beclin 1-PI3K complexes," Autophagy, vol. 5, no. 4, pp. 534-536, 2009.

[14] K. Matsunaga, T. Saitoh, K. Tabata et al., "Two Beclin 1binding proteins, Atg14L and Rubicon, reciprocally regulate autophagy at different stages," Nature Cell Biology, vol. 11, no. 4, pp. 385-396, 2009.

[15] H. D. Xu and Z. H. Qin, "Beclin 1, Bcl-2 and autophagy," Advances in Experimental Medicine and Biology, vol. 1206, pp. 109-126, 2019.

[16] V. Hubert, A. Peschel, B. Langer, M. Gröger, A. Rees, and R. Kain, "LAMP-2 is required for incorporating syntaxin-17 into autophagosomes and for their fusion with lysosomes," Biology Open, vol. 5, no. 10, pp. 1516-1529, 2016.

[17] E. L. Eskelinen, "Roles of LAMP-1 and LAMP-2 in lysosome biogenesis and autophagy," Molecular Aspects of Medicine, vol. 27, no. 5-6, pp. 495-502, 2006.

[18] S. Liu, C. Sarkar, M. Dinizo et al., "Disrupted autophagy after spinal cord injury is associated with ER stress and neuronal cell death," Cell Death \& Disease, vol. 6, no. 1, 2015.

[19] J. Huang, V. Canadien, G. Y. Lam et al., "Activation of antibacterial autophagy by NADPH oxidases," Proceedings of the National.Academy of Sciences of the United States of America, vol. 106, no. 15, pp. 6226-6231, 2009. 
[20] R. Scherz-Shouval, E. Shvets, and I. Elazar, "Oxidation as a post-translational modification that regulates autophagy," Autophagy, vol. 3, no. 4, pp. 371-373, 2007.

[21] G. Filomeni, D. De Zio, and F. Cecconi, "Oxidative stress and autophagy: the clash between damage and metabolic needs," Cell Death and Differentiation, vol. 22, no. 3, pp. 377-388, 2015.

[22] A. Lau, S. A. Whitman, M. C. Jaramillo, and D. D. Zhang, "Arsenic-mediated activation of the Nrf2-Keap1 antioxidant pathway," Journal of Biochemical and Molecular Toxicology, vol. 27, no. 2, pp. 99-105, 2013.

[23] H. Zhao, Y. Wang, Y. Liu et al., "ROS-induced hepatotoxicity under cypermethrin: involvement of the crosstalk between $\mathrm{Nrf} 2 / \mathrm{Keap} 1$ and NF- $\kappa \mathrm{B} / \mathrm{i} \kappa \mathrm{B}-\alpha$ pathways regulated by proteasome," Environmental Science \& Technology, vol. 55, no. 9, pp. 6171-6183, 2021.

[24] X. Y. Wang, Z. Y. Wang, Y. S. Zhu, S. M. Zhu, R. F. Fan, and L. Wang, "Alleviation of cadmium-induced oxidative stress by trehalose via inhibiting the Nrf2-Keap1 signaling pathway in primary rat proximal tubular cells," Journal of Biochemical and Molecular Toxicology, vol. 32, no. 1, article e22011, 2018.

[25] T. Jiang, B. Harder, M. Rojo de la Vega, P. K. Wong, E. Chapman, and D. D. Zhang, "P62 links autophagy and Nrf2 signaling," Free Radical Biology \& Medicine, vol. 88, pp. 199-204, 2015.

[26] A. Sureshbabu, S. W. Ryter, and M. E. Choi, "Oxidative stress and autophagy: crucial modulators of kidney injury," Redox Biology, vol. 4, pp. 208-214, 2015.

[27] N. Fujiwara, T. Usui, T. Ohama, and K. Sato, "Regulation of Beclin 1 Protein Phosphorylation and Autophagy by Protein Phosphatase 2A (PP2A) and Death-associated Protein Kinase 3 (DAPK3)," The Journal of Biological Chemistry, vol. 291, no. 20, pp. 10858-10866, 2016.

[28] Z. Papackova, H. Dankova, E. Palenickova, L. Kazdova, and M. Cahova, "Effect of short- and long-term high-fat feeding on autophagy flux and lysosomal activity in rat liver," Physiological Research, vol. 61, pp. S67-S76, 2012.

[29] L. Chakrabarti, J. Eng, N. Ivanov, G. A. Garden, and A. R. La Spada, "Autophagy activation and enhanced mitophagy characterize the Purkinje cells of pcd mice prior to neuronal death," Molecular Brain, vol. 2, no. 1, p. 24, 2009.

[30] S. Pankiv, T. H. Clausen, T. Lamark et al., "p62/SQSTM1 Binds Directly to Atg8/LC3 to Facilitate Degradation of Ubiquitinated Protein Aggregates by Autophagy," The Journal of Biological Chemistry, vol. 282, no. 33, pp. 24131-24145, 2007.

[31] A. Goode, K. Butler, J. Long et al., "Defective recognition of LC3B by mutant SQSTM1/p62 implicates impairment of autophagy as a pathogenic mechanism in ALS-FTLD," Autophagy, vol. 12, no. 7, pp. 1094-1104, 2016.

[32] C. T. Tan, H. C. Chang, Q. Zhou et al., "MOAP-1-mediated dissociation of p62/SQSTM1 bodies releases Keap1 and suppresses Nrf2 signaling," EMBO Reports, vol. 22, no. 1, 2021.

[33] W. Yue, A. Hamaï, G. Tonelli et al., "Inhibition of the autophagic flux by salinomycin in breast cancer stem-like/progenitor cells interferes with their maintenance," Autophagy, vol. 9, no. 5, pp. 714-729, 2013.

[34] Y. Chen and L. Yu, "Recent progress in autophagic lysosome reformation," Traffic, vol. 18, pp. 358-361, 2017.

[35] W. Xu, L. J. Xin, L. Song, and K. Zhang, "Sphingolipid degradation by leishmania major is required for its resistance to acidic ph in the mammalian host," Infection and immunity, vol. 79, pp. 3377-3387, 2011.

[36] S. Chikte, N. Panchal, and G. Warnes, "Use of LysoTracker Dyes: a flow cytometric study of autophagy," Cytometry. Part A, vol. 85, pp. 169-178, 2014.

[37] A. Pierzyńska-Mach, P. A. Janowski, and J. W. Dobrucki, "Evaluation of acridine orange, LysoTracker Red, and quinacrine as fluorescent probes for long-term tracking of acidic vesicles," Cytometry. Part A, vol. 85, no. 8, pp. 729-737, 2014.

[38] M. P. Thomé, E. C. Filippi-Chiela, E. S. Villodre et al., "Ratiometric analysis of acridine orange staining in the study of acidic organelles and autophagy," Journal of Cell Science, vol. 129, pp. 4622-4632, 2016.

[39] M. J. Sousa, F. Azevedo, A. Pedras et al., "Vacuole-mitochondrial cross-talk during apoptosis in yeast: a model for understanding lysosome-mitochondria-mediated apoptosis in mammals," Biochemical Society Transactions, vol. 39, no. 5, pp. 1533-1537, 2011.

[40] M. E. Orr and S. Oddo, "Autophagic/lysosomal dysfunction in Alzheimer's disease," Alzheimer's Research \& Therapy, vol. 5, p. 53, 2013.

[41] B. Haidar, R. S. Kiss, L. Sarov-Blat et al., "Cathepsin D, a Lysosomal Protease, Regulates ABCA1-mediated Lipid Efflux," The Journal of Biological Chemistry, vol. 281, no. 52, pp. 3997139981, 2006.

[42] F. C. Sigloch, M. Tholen, A. Gomez-Auli, M. L. Biniossek, T. Reinheckel, and O. Schilling, "Proteomic analysis of lung metastases in a murine breast cancer model reveals divergent influence of CTSB and CTSL overexpression," Journal of Cancer, vol. 8, no. 19, pp. 4065-4074, 2017.

[43] T. Aki, T. Funakoshi, K. Unuma, and K. Uemura, "Impairment of autophagy: from hereditary disorder to drug intoxication," Toxicology, vol. 311, pp. 205-215, 2013.

[44] S. J. Moon, J. Jhun, J. Ryu et al., "The anti-arthritis effect of sulforaphane, an activator of Nrf2, is associated with inhibition of both $\mathrm{B}$ cell differentiation and the production of inflammatory cytokines," PLoS One, vol. 16, no. 2, 2021.

[45] D. H. Lee, J. S. Park, Y. S. Lee et al., "SQSTM1/p62 activates NFE2L2/NRF2 via ULK1-mediated autophagic KEAP1 degradation and protects mouse liver from lipotoxicity," Autophagy, vol. 16, no. 11, pp. 1949-1973, 2020.

[46] L. H. Wang, G. J. Qu, Y. D. Gao et al., "A small molecule targeting glutathione activates Nrf2 and inhibits cancer cell growth through promoting Keap-1S-glutathionylation and inducing apoptosis," RSC Advances, vol. 8, no. 2, pp. 792 804, 2018.

[47] K. Taguchi, N. Fujikawa, M. Komatsu et al., "Keap1 degradation by autophagy for the maintenance of redox homeostasis," Proceedings of the. Nationall Academy of Sciences of the United States of America, vol. 109, no. 34, pp. 13561-13566, 2012.

[48] A. Jain, T. Lamark, E. Sjøttem et al., “p62/SQSTM1 Is a Target Gene for Transcription Factor NRF2 and Creates a Positive Feedback Loop by Inducing Antioxidant Response Elementdriven Gene Transcription," The Journal of Biological Chemistry, vol. 285, no. 29, pp. 22576-22591, 2010.

[49] M. Komatsu, H. Kurokawa, S. Waguri et al., "The selective autophagy substrate p62 activates the stress responsive transcription factor Nrf2 through inactivation of Keap1," Nature Cell Biology, vol. 12, no. 3, pp. 213-223, 2010. 
[50] H. L. Yang, C. H. Tsai, S. Shrestha, C. C. Lee, J. W. Liao, and Y. C. Hseu, "Coenzyme $\mathrm{Q}_{0}$, a novel quinone derivative of Antrodia camphorata, induces ROS-mediated cytotoxic autophagy and apoptosis against human glioblastoma cells in vitro and in vivo," Food and Chemical Toxicology, vol. 155, 2021.

[51] Y. He, Y. Shi, H. Huang et al., "Chrysin induces autophagy through the inactivation of the ROS-mediated Akt/mTOR signaling pathway in endometrial cancer," International Journal of Molecular Medicine, vol. 48, no. 3, p. 172, 2021.

[52] Y. Hu, G. Zhao, L. Qin et al., "Trans,trans-2,4-decadienal induces endothelial cell injury by impairing mitochondrial function and autophagic flux," Food \& Function, vol. 12, no. 12, pp. 5488-5500, 2021.

[53] X. Zhu, S. Liu, Z. Cao et al., "Higenamine mitigates interleukin- $1 \beta$-induced human nucleus pulposus cell apoptosis by ROS-mediated PI3K/Akt signaling," Molecular and Cellular Biochemistry, vol. 476, no. 11, pp. 3889-3897, 2021.

[54] T. Martins-Marques, A. Rodriguez-Sinovas, and H. Girao, "Cellular crosstalk in cardioprotection: where and when do reactive oxygen species play a role?," Free Radical Biology \& Medicine, vol. 5849, 2021.

[55] Q. Gao, "Oxidative stress and autophagy," in Autophagy: Biology and Diseases, vol. 1206 of Advances in Experimental Medicine and Biology, , pp. 179-198, Springer, 2019.

[56] S. Kassis, M. Grondin, and D. A. Averill-Bates, "Heat shock increases levels of reactive oxygen species, autophagy and apoptosis," Biochimica et Biophysica Acta (BBA)-Molecular Cell Research, vol. 1868, 2021.

[57] W. R. Wilson and M. P. Hay, "Targeting hypoxia in cancer therapy," Nature Reviews. Cancer, vol. 11, no. 6, pp. 393-410, 2011. 